\title{
The Active Eye: Software for the demonstration of event perception phenomena on the Macintosh
}

\author{
GEOFFREY P. BINGHAM \\ Indiana University, Bloomington, Indiana
}

The Active Eye Stack (Software)

By Michael I. Mills \& William Schiff. 1989, Hillsdale, NJ: Erlbaum. \$99.00.

\section{Active Eye Stack Guidebook \\ By Michael I. Mills \& William Schiff. 1988, Hillsdale, NJ: Erlbaum. 152 pp. $\$ 10.00$.}

A great difficulty in studying human visual perception is to convince the uninitiated that there are problems to be profitably investigated beyond the confines of an armchair. The intimate and remarkably robust and effective character of our perceptual abilities fosters an implicit assumption of privileged access and a false sense of understanding. If, perhaps, the essential nature of perception is not immediately accessible to our intelligence, it apparently seems that at least the basic phenomena of interest should be accessible and familiar to everyone and uncontroversial. In fact, however, some of the most profound phenomena in perception are ones about which we have the least intuition. Most of the phenomena associated with motion are of this sort. Event perception is not open to introspective examination and analysis. Early structuralists employed thoroughgoing introspection to relegate event phenomena to the uninspiring and uninformative category of "gray blur." More recently, the lack of introspective access has even been used to target experimental manipulations of event phenomena. An esteemed investigator of event perception, James Todd, has noted in conversation his use of an experimental participant's ignorance as a criterion for evaluating experimental tasks in event perception; the more absurd the subject's account, the more illuminating and reliable Todd expects the data to be. Further illustration of the difficulty is provided by the fact that participants are often quite astute in performing event perception tasks that, in advance, they believe themselves unable to perform, such as the judging of amounts of lifted weight from a patch-light video display (Bingham, 1987; Runeson \& Frykholm, 1981, 1983).

Correspondence should be addressed to Geoffrey P. Bingham, Department of Psychology, Indiana University, Bloomington, IN 47405.
Given this common lack of native insight concerning the nature of event perception and its phenomena, classroom demonstrations are essential both to convey an understanding of the subject matter and to inspire interest. Before microcomputers became readily available, movies and video tapes were the only means of illustrating a broad variety of effects in the lecture hall. The obvious drawbacks were the cost and difficulties associated with the need to obtain, keep, and organize a library of films. Also, films illustrating many important phenomena have remained unavailable. For those that have been available, a significant limitation has been the inability to vary parameters in controlling displays so that the lecturer or students can explore the conditions that determine various effects. The upshot has been that a true appreciation of both the phenomena and the problems of event perception has been reserved largely for those who venture into the laboratory.

In the late 1980s, the rapidly increasing availability and use of powerful, yet affordable, microcomputers brought the promise of a solution to this problem. This was especially true of the Macintosh, with its powerful and sophisticated graphics capabilities. Two developments in Macintosh software preceded and enabled the creation of a software package designed to meet the needs of the lecturer in event perception. The first was the development of HyperCard, which is a high-level language for programming hierarchically organized presentations on the Macintosh. The second was the development of VideoWorks, which is a software package for creating animations on the Macintosh. Using HyperCard and VideoWorks in combination, Michael I. Mills, in collaboration with William Schiff, programmed The Active Eye to provide hierarchically accessed information about research in event perception, accompanied by animations illustrating the various phenomena and effects. In 1988, Erlbaum Associates began marketing The Active Eye, subsequently reducing the price to affordable levels. The result, though not a complete solution, does much to alleviate the need for diverse and readily available, interactive demonstrations in event perception.

The Active Eye includes a 152-page guidebook, together with Macintosh software for approximately 50 different 
animated demonstrations accompanied by explanatory and reference materials. The guidebook is largely the product of William Schiff. A past student of J. J. Gibson's, and the author of a well-known perception text, Schiff is perhaps best known for his research on looming, impeding collision and time-to-contact phenomena in optic flow. The guidebook overlaps with and elaborates on textual material contained within the Active Eye software. Part I contains introductory material, including instructions for running the software, which, like most Macintosh applications, is fairly self-explanatory once loaded into the machine and started up. The remainder of the guidebook contains units corresponding to the various demonstrations, each of which provides a brief theoretical and historical background; a description of the respective effects, including illustrative images from the animations; instructions for manipulating the animation; a bibliography of related research publications; some textbook references, including relevant page numbers; and finally, a listing of other related demonstrations within The Active Eye. The guidebook is nicely formatted and well written. Representative material is presented from the work of Julian Hochberg, James Gibson, Hans Wallach, Irvin Rock, Gunnar Johansson, Adelbert Ames, James Cutting, Albert Michotte, Fritz Heider, Richard Haber, William Schiff, and David Lee, among others, and from the perspective of Gestalt, Information Processing, Gibsonian, and Helmholzian approaches to perceptual psychology. Absent are references to recent computational, and in particular, connectionist, approaches to perceptual problems. On the other hand, the guidebook is concerned less with attempts to model perceptual processes than with presentation of the most prominent phenomena discovered within the field. With this observation, it is fair to say that a truly representative sample of the field of perceptual psychology is presented, with special reference to event perception.

The software was programmed by Michael Mills, a perceptual psychologist who is currently working for Apple in California. At the time of its creation, The Active Eye innovated the combination of Hypercard and VideoWorks. Some minor, functionally irrelevant bugs remain in the package as a result of difficulties associated with interfacing the two applications. Overall, the package reflects the structure common to all Macintosh software. The organization of files in the Macintosh is hierarchical, with files grouped into folders that may be opened to reveal items at that node organized at the next level down in the hierarchy. Once the Active Eye software has been loaded onto one's hard disk, the Active Eye folder should reside in the Hypercard folder alongside Hypercard and the Hypercard Home stack. (An Active Eye button can be installed on the home card.) The Active Eye folder occupies about $3,300 \mathrm{~K}$ on the disk and contains a variety of files, including the main Active Eye stack, a help file, the VideoWorks driver and accelerator, and the animations themselves. (The VideoWorks accelera- tor allows smooth motion in complex animations.) The Active Eye is entered primarily through the main Active Eye stack. This stack resides at the top of the Active Eye hierarchy and consists of a series of seven menu cards, which can be paged though in any order by clicking the mouse with the cursor positioned on the appropriate buttons located on the menu cards. Similarly, a button for the help file exists on almost all the cards, so that the help file, which contains the information in the introductory section of the guidebook, can be accessed from anywhere within The Active Eye. In addition to the help file button, each menu card in the main stack contains two buttons for each of eight demonstrations.

The first button for each demonstration opens a synopsis card that contains a brief description of the background, elaborated in the guidebook, references, and a list of related demonstrations. From the synopsis card, one can either return to the main stack or move directly to the animation. The second button for each demonstration in the main stack also opens the animation itself.

With a couple of exceptions to be described subsequently, one of two types of toggle switch allows manipulation of each animation. The first type of toggle switch allows the animation to be played either forward or backward, one frame at a time. The second type of toggle switch allows the frame rate to be either increased or decreased, with the frames per second displayed in a small window. (A slight problem here is that the frame rate is displayed only for a second after the switch has been toggled, leaving the frame rate window blank most of the time. If unknown, the current frame rate can only be assessed by changing the rate! One has to pay close attention to catch the new rate.) A given animation includes only one of the two types of toggle switches. The attentive reader will see immediately that The Active Eye allows only rather limited interaction with the displays. The limitations are especially disappointing in specific instances and of much less concern in others.

One of the first demonstrations happens to be one of the more disappointing. The animation illustrates apparent motion between two black dots on a white field. In this case, the frame rate can be altered. However, the distance between the dots or the relative brightness of the dots cannot be altered. Thus, Korte's laws, which are discussed in the guidebook, cannot really be explored. Another even more disappointing limitation appears in a demonstration of Michotte's collision displays. In this instance, only single frame stepping is enabled, which is essentially useless. The lack of an ability to vary the timing in this case renders the demonstration completely ineffective, since the effect of interest is a change in the perceived nature of the event (e.g., from collision to launching) with a change in the timing. Hume himself could see on the billiard table displays like that in The Active Eye. True, he could not step through his "displays" frame by frame, but had he been able to do so, it might have served only to strengthen his opinions and analysis. 
The variation in perceived significance with variation in the timing of the kinematics brings home Michotte's contradiction of Hume's analysis.

These demonstrations aside, however, the limited nature of the animation controls is not a problem with the vast majority of the displays, which are entirely effective, wonderful to see, and great fun to manipulate and explore. Many of the demonstrations can be classed as illustrating closely related types of effects. For instance, seven demonstrations illustrate Johansson's and Cutting's work on "frames of reference" that arise in the relative motions of points in a plane. Another five demonstrations illustrate variations of Kanisza-type illusory contour figures in motion. Still yet another six or seven displays explore visual persistence and so-called "anorthoscopic form" perception as investigated by Rock and Hochberg. These demonstrations include the barber pole effect, various successive visual sampling displays, and some tachistoscopic letter and word recognition demonstrations. Also illustrated is the Ames trapezoidal window (seen from either the front or the top), the kinetic depth effect, the Ternus effect, and a Johansson point-light walker. An oddity in the point-light walker display occurs when two points pass through one another as their paths of motion intersect. Both points gradually disappear and then reappear as they pass. Yet the perceived significance of the display is notably robust despite this anomalous type of occlusion.

A second "biological motion" animation, illustrating the ability to recognize the gender of a person in a patchlight display, is slightly disappointing for its failure to illustrate Cutting's "center of moment" analysis, which is discussed in the guidebook. The implied point of the demonstration is that gender can be judged in the absence of the information hypothesized by Cutting. Indeed, gender is usually identified correctly in the display provided. Nevertheless, since Cutting's analysis was discussed at such length in the guidebook, an illustration of the displays analyzed by Cutting would have been useful.

In addition to Johansson's "biological motion" displays, a digitized version of the original Fritz Heider social interaction film is included. A section on "structure from motion" includes random texture displays very similar to the original films made by J. J. Gibson and George Kaplan in the late 1960s. Separated surfaces appear with motion, but the separation collapses an instant after motion ceases. Stepping the displays causes them to pop out and collapse in succession. The effect is stunning and enthralling. The displays include a moving textured surface of indefinite extent seen as if through a window, a smaller surface moving in front of a larger background surface, and an elongated textured surface rotating in front of a background surface in a fashion imitating the trapezoidal window. Of course, no ambiguity exists in the motion of the surface in this instance.

There is a substantial selection of optic flow demonstrations and tutorials. These include an optic flow field display corresponding to motion over a horizontal plane, a motion gradient display simulating the passing of a line of fence posts extending into the distance as seen through a window, a series of displays illustrating the flow associated with decelerative approach to a surface, a series of displays illustrating the flow associated with constant velocity approach to a surface with "looming," a timeto-contact experiment, and a tutorial on the radial flow associated with approach to a surface.

A number of the demonstrations include tutorials in which the analysis of a given animation is presented via text and diagrams. For instance, the cycloid generated by the motion of a dot on the rim of a rolling wheel is shown as part of the discussion in the first "frames of reference" demonstration. The radial motion tutorial includes some introductory material and then allows the user to determine the initial distance of an approaching surface, its height, and the constant step size of its approach. Four different presentations of the resulting approach may then be examined. They include a side view showing rays successively projected from the top of the surface to the back of an outsized eye, a head-on view of the approach, and a graph showing the exponential changes in the projection produced by linear changes in the position of the surface. The tutorial is very useful for illustrating the problem of mapping from changes in an event to optical changes.

The time-to-collision experiment allows the user to replicate Schiff and Detwiler (1979). In the display, a surface is seen to approach the screen surface from varying distances and at various (constant) velocities. At a point along the trajectory preceding contact with the screen, the surface disappears. The observer's task is to click the mouse on a timer at the moment when the surface would hit the screen should it continue its trajectory. (A disclaimer in the introduction to the guidebook notes that absolute times may vary between different Macintosh models and therefore should be regarded as approximate.) After each trial, the actual and judged times-to-contact are shown. The total number of trials may be determined by the user. After the last trial, the data are tabulated and displayed both in a table and in a plot of mean judged versus actual times-to-contact for three actual time-to-contact values. (No information about variability or error bars is provided.) I found that the task required a small amount of practice, after which I have regularly reproduced Schiff and Detwiler's original results - that is, increasing underestimation with increasing time to contact.

There are a number of demonstrations that I find less useful. One, which sets a contour map of a face in motion, seems to be included mostly to advertise the contour drawing software used to create the images. A couple of others are included primarily as jokes. Another, called the "Transformer," distorts a couple of faces in unspecified ways and remains unexplained in the guidebook. Still another animation simply rotates the block figures commonly used in the mental rotation experiments. A package written by Mills for the Ontario Science Centre on judging sports events is included in The Active Eye as 
a unit on "expert perceiving." Although it is interesting as a tutorial on judging competitive gymnastics, it does not seem to be informative in any direct way about problems in perceptual learning. Perhaps I am missing something, but it seems to have been included in case someone should have a bright idea about what to do with it. In the guidebook, reference is made to the Chase and Simon (1973) article on chess masters as experts in remembering legal chess configurations. But the discussion of chunking, powerful perceptual "hardwiring," and mental formulas is not very illuminating with respect to expertise in event perception.

Whether or not the user finds the latter demonstrations to be of value, the remaining corpus certainly makes this package a valuable asset to have in one's arsenal of teaching resources and a package certainly worth the fairly modest price. The authors are to be commended for their ground-breaking efforts. There is some possibility that the authors may rejoin their efforts to produce an expanded and more powerful version of The Active Eye, one that allows increased control over the animation parameters as well as enables the user to design displays and experiments. The design and programming effort required by such an enterprise is monumental. We can only cross our fingers and hope. Meanwhile, we can appreciate the extant demonstration capabilities provided by The Active Eye.
On the technical side, the software requires a Mac Plus or SE with 2.5MB RAM or a Mac II with 2MB RAM, each together with a 20MB hard disk, System 4.3 or greater, Finder 6.0 , and HyperCard 1.2 or greater. The VideoWorks accelerator required to run some of the animations comes with the package. The 152-page guidebook may also be purchased separately. A final note: My oneand-a-half-year-old copy contains a fair number of typos. For example, the kinetic depth effect is attributed to Wallach, O'Connell, and Neisser! I understand that these have been fixed in subsequent copies.

\section{REFERENCES}

Bingham, G.P. (1987). Scaling and kinematic form: Further investigations on the visual perception of lifted weight. Joumal of Experimental Psychology: Human Perception \& Performance, 13, 155-177.

Chase, W., \& Simon, H. (1973). Perception in chess. Cognitive Psychology, 4, 55-81.

Runeson, S., \& Frykholm, G. (1981). Visual perception of lifted weight. Jourmal of Experimental Psychology: Human Perception \& Performance, 7, 733-740.

RUNESON, S., \& Frykholm, G. (1983). Kinematic specification of dynamics as an informational basis for person and action perception: Expectation, gender, recognition, and deceptive intention. Journal of Experimental Psychology: General, 112, 585-615.

SCHIFF, W., \& Detwiler, M. L. (1979). Information used in judging impending collisions. Perception, 8, 647-658. 